\title{
Effects of the Corticosteroids Treatment on the Nucleic Acid Contents of Tumor Bearing Rat Lymphoid Tissues
}

\author{
Shigemi ASAZUMA \\ Department of Obstetrics and Gynecology, School of Medicine, Kanazawa University
}

(Director : Prof. Dr. Fumio Akasu)

Under the influence of corticosteroids, it is well known that lymphnode falls into marked atrophy. Akasu and Tateno (1963) observed experimentaly the rat lymphnodes, pretreated with cortisone, seldom attacked with the metastasis of rat ascites hepatoma, and they assumed the possibility of arrest of cancer metastasis in man.

In previous study it has been found in rat lymphnodes that the decrease of DNA contents and the rise of the ratio of RNA to DNA contents were caused by treatment with cortisone. The purpose of the present study is to investigate the changes, induced by cortisone, which probably had prevented the lymphnodes from the metastasis of implanted ascites hepatoma, by means of determination of the nucleic acid contents in the lymphoid tissues.

Forty female rats, weighing between 80 and 120g., were divided in half. Each animal of the one half received $2.5 \mathrm{mg}$. of cortisone acetate, injected subcutaneously, each day for 10 days (Treated group). Each of the other half received $0.1 \mathrm{ml}$. of physiological salt solution, instead of cortisone, injected similarly (Control group).

Twenty animals in all, ten animal apiece picked out of the two groups, got ascites hepatoma AH 130 implated intraperitoneally on the seventh day after the first injection of cortisone or salt solution. Then, twenty-four hours after the final injection, all animals were killed. Twenty microcurie of ${ }^{32} \mathrm{P}$ solution was subcutaneouly injected into each animal at six hours before the sacrifice.

After the sacrifice of the animals, thymus, lymphnodes and spleen were excised for the measurement of the nucleic acid contents and the ${ }^{32} \mathrm{P}$ amounts incorporated into the nucleic acids. Throughout this experiment, ribonucleic acids (RNA) were determined by the color reaction of its pentose with orcine and deoxyribonucleic acids (DNA) by the reaction with diphenylamine, after the separation of RNA and DNA by the method based on Schmidt and Thannhauser procedure.

The results of the experiment are summarized as follows:

(1) The implantation of ascites hepatoma carried the thymus into atrophy, in which RNA and DNA contents were decreased and the incorporation of ${ }^{32} \mathrm{P}$ into the nucleic acids were remarkably inhibited. These changes occurred in the thymus were promoted by the simultaneous treatment with cortisone.

$$
\text { Vol. } 41 \text { No. } 6
$$


(2) Under the influence of the implanted tumor, untreated lymphnodes swelled in size, attended by an increase of RNA contents and a lowering of the ratio of RNA to DNA contents. In treated lymphnodes, however, these changes except for a little hypertrophy were not caused by the implantation of the tumor.

Reversely, under the influence of the implantation, the increase of DNA contents and the elevation of the ratio of RNA to DNA contents were found in treated lymphodes. This elevation of ratio, induced by cortisone, seems to have some relation to the prevention of the metastasis.

(3) The incorporation of ${ }^{32} \mathrm{P}$ into the spleen RNA was promoted by the implantation of the tumor unrelated to the treatment. In this experiment, however, neither the treatment with cortisone nor the implantation of the tumor affected the nucleic acid contents of the spleen.

(pp. 776〜790) 